\title{
ERRATUM: Exonic duplication CNV of NDRG1 associated with autosomal-recessive HMSN-Lom/CMT4D
}

Yuji Okamoto MD, PhD, Meryem Tuba Goksungur MD, Davut Pehlivan MD, Christine R. Beck PhD, Claudia Gonzaga-Jauregui PhD, Donna M. Muzny MS, Mehmed M. Atik, Claudia M.B. Carvalho PhD, Zeliha Matur MD, Serife Bayraktar MD, Philip M. Boone PhD, Kaya Akyuz BS, Richard A. Gibbs PhD, Esra Battaloglu PhD, Yesim Parman MD and James R. Lupski MD, PhD

Genet Med advance online publication, October 17, 2013; doi:10.1038/gim.2013.155

In Table 1 in the version of this article initially published, units in the Sensory NCSs row were incorrectly printed as "Amp (mV)." The correct units are "Amp $(\mu \mathrm{V})$." The corrected table appears below, and the error has been corrected in all versions of the article. The publisher regrets the error.

Table 1 Sensory and motor nerve conduction studies of three patients and one healthy member of the family

Patient

(age in years)

(age in years)

Motor NCSs

BAB3724 (30)

dI $v$ Amp

R axillary

BAB5658 (28)

$R$ musculocutaneus

II-2

Amp

$\begin{array}{lllll}14.5 & \mathrm{~N} / \mathrm{A} & 5.8 & \mathrm{~N} / \mathrm{A} & \mathrm{N} / \mathrm{A}\end{array}$

(mV)

$\mathrm{N} / \mathrm{A}$
$12.9 / 11.1$

R median

R ulnar

$\mathrm{R}$ common peroneal

R tibialis

$7.1 \quad \mathrm{~N} / \mathrm{A}$

0.9

NR NR NR

$N R \quad N R \quad N R$

N/A N/A

3.2

NR NR

$\begin{array}{llllll}N R & N R & N R & 4.7 & 49 & 11.2 / 10.3\end{array}$

Sensory NCSs

dL $\quad \mathrm{V} \quad \mathrm{Amp} \quad \mathrm{dL} \quad \mathrm{V}$ Amp $\mathrm{dL}$

$R$ median

(ms) $\quad(\mathrm{m} / \mathrm{s}) \quad(\mu \mathrm{V}) \quad(\mathrm{ms}) \quad(\mathrm{m} / \mathrm{s})$

R ulnar

$\mathrm{R}$ radial

R superficial peroneal

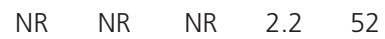

NR NR

NR N/A N/A

$\begin{array}{lllll}N R & N R & N R & 2.3 & 53\end{array}$

L superficial peroneal

R sural

N/A $\quad$ N/A $\quad$ N/A $2.3 \quad 53$

$\begin{array}{lllll}N R & N R & N R & 2.7 & 53\end{array}$

$\begin{array}{lllll}\text { N/A } & \text { N/A } & \text { N/A } & 2.7 & 52\end{array}$

L sural
N/A

$(\mu \mathrm{V})$

$(\mu \mathrm{V})$

17.0

9.4

N/A

8.6

BAB4149 (24)

\begin{tabular}{lll}
\hline \multicolumn{3}{c}{ II-4 } \\
\hline $\mathrm{dL}$ & $\mathrm{v}$ & \\
&
\end{tabular}

dL $\quad \mathrm{V} \quad \mathrm{Amp}$

$\begin{array}{lll}\text { (ms) } & (\mathrm{m} / \mathrm{s}) \quad(\mathrm{mV})\end{array}$

$\begin{array}{lll}15.2 & \mathrm{~N} / \mathrm{A} & 0.2\end{array}$

$\begin{array}{lll}7.2 \quad \mathrm{~N} / \mathrm{A} \quad 1.5 & \end{array}$

NR

$N R$

NR NR

NR

NR NR

$\mathrm{dL} \quad \mathrm{V}$

(ms) (m/s)

NR NR

NR NR

NR

NR NR

NR NR

4.6

N/A N/A

$20.8 \quad N R \quad N R$

23

N/A N/A

$N R \quad 2.8$
$N R$

BAB4152 (17)

\begin{tabular}{c} 
II-7 \\
\hline $\mathrm{V}$
\end{tabular}

Normal values

(N/A

amp, amplitude; $\mathrm{CV}$, condulthy member. bRecorded from extensor digitorum brevis and tibialis anterior muscles.

ERRATUM: Processes and preliminary outputs for identification of actionable genes as incidental findings in genomic sequence data in the Clinical Sequencing Exploratory Research Consortium

Jonathan S. Berg MD, PhD, Laura M. Amendola MS, Christine Eng MD, Eliezer Van Allen MD, Stacy W. Gray MD, AM, Nikhil Wagle MD, Heidi L. Rehm PhD, Elizabeth T. DeChene MS, Matthew C. Dulik PhD, Fuki M. Hisama MD, Wylie Burke MD, PhD, Nancy B. Spinner PhD, Levi Garraway MD, PhD, Robert C. Green MD, MPH, Sharon Plon MD, PhD, James P. Evans MD, PhD and Gail P. Jarvik MD, PhD and the members of the CSER Actionability and Return of Results Working Group

Genet Med 15: 860-867; advance online publication, November 6, 2013; doi:10.1038/gim.2013.133

In the initially published version of this article, the advance online publication date was incorrect. The correct date is November 6, 2013. The publisher regrets the error. 